\title{
Marked socioeconomic inequalities in hip fracture incidence rates during the Bone and Joint Decade (2000-2010) in Portugal: age and sex temporal trends in a population based study
}

\author{
Carla Maria Oliveira, ${ }^{1,2,3,4,5}$ Sandra Maria Alves, ${ }^{1,2,3}$ Maria Fátima Pina 1,2,4,6,7
}

Author affiliations

${ }^{1}$ i3S - Instituto de Investigação e Inovacãa em Saúde, Universidade do Porto, Porto, Pórtugal

${ }^{2}$ INEB - Instituto de Engenharia Biomédica, Universidade do Porto, Porto, Portugal ${ }^{3}$ ESTSP/IPP_Escola Superior de Tecnologia da saúde do Porto Instituto Politécnico do Porto, Vila Nova de Gaia, Portugal

${ }^{4}$ FMUP_Faculdade de Medicina da Universidade do Porto, Porto, Portugal

${ }^{5}$ ISPUP — Instituto de Saúde Pública da Universidade do Porto, Porto, Portugal

${ }^{6} \mathrm{ICICT} /$ FIOCRUZ—Instituto de Comunicação e Informacão em Saúde/Fundação Oswaldo Cruz, Rio de Janeito, Brazi

${ }^{7}$ CARTO-FEN/UERJ—Departamento de Engenharia Gartográfica Eaculdade de Engenharia da Francisco Xavier, Rio de Janeiro—RJ, Brazil

\section{ABSTRACT}

Background Socioeconomic factors may influence changes in hip fracture (HF) incidence over time. We analysed HF temporal trends during the Bone and Joint Decade in Portugal (BJD-Portugal), 2000-2010, by regional socioeconomic status (SES), sex and age.

Methods We selected registers of patients aged $50+$ years with HF (International Classification of Diseases, V.9-Clinical Modification, ICD9-CM) caused by traumas of low/moderate energy, from the National Hospital Discharge Database. Annual time series of age-specific incidence rates were calculated by sex and regional SES (deprived, medium, affluent). Generalised additive models were fitted to identify shape/turning points in temporal trends.

Results We selected 96905 HF (77.3\% in women). Women were older than men at admission $(81.2 \pm 8.5$ vs $78.2 \pm 10.1$ years-old, $p<0.001)$. For women 65 79 years, a continuously decreasing trend (1.7\%/year) only in affluent and increasing trends (3.3-3.4\%/year) after 2006/2007 in medium and deprived was observed. For men, trends were stable or increased in almost all age/SES groups (only two decreasing periods). For the oldest women, all SES present similar trends: turning points around 2003 (initiating decreasing periods: 1.8-2.9\%/year) and around 2007 (initiating increasing periods: $3.7-3.3 \% /$ year).

Conclusions There were SES-sex-age inequalities in temporal trends during BJD-Portugal: marked SES inequalities among women aged $65-79$ years (a persistent, decreasing trend only in the affluent) vanished among the oldest women; the same was not observed in men, for them, there were almost no declining periods; women aged $\geq 80$ years, presented increasing trends around 2007, as in most deprived/age/ sex groups. Despite some successful periods of decreasing trends, incidence rates did not improve overall in almost all age groups and both sexes.

\section{INTRODUCTION}

Hip fractures (HF), some of the most preventable causes of disabilities in the elderly, ${ }^{1}$ are a major public health problem because of their association with high morbidity and mortality rates, and their economic impact on individuals and societies. ${ }^{2}$ Therefore, HP were included among the disorders prioritised by the Bone and Joint Decade (BJD) initiative. This initiative resulted from the concern of healthcare professionals over the significant impact of these disorders on society, the healthcare system and individuals. Launched in 2000 and running until 2010, its primary aim was to promote advances in the knowledge, diagnosis and treatment of musculoskeletal disorders. The goal of the BJD was: "to improve the health-related quality of life for people with musculoskeletal disorders throughout the world by raising awareness and promoting positive actions to combat the suffering and costs to society, associated with musculoskeletal disorders" ${ }^{3}$ More than 60 countries, including Portugal, signed this initiative ${ }^{4}$ and made commitments to reduce HF incidence. The BJD was renewed for the decade 2010-2020 as a global alliance for musculoskeletal health. ${ }^{5} 6$

It is known that socioeconomic factors are determinants for disparities in health behaviours, and incidence of diseases and mortality; ${ }^{7}$ an inverse association between several health outcomes and socioeconomic status (SES) has been reported: deprived SES individuals, or regions, present higher morbidity and mortality rates in general. ${ }^{7}$ The inverse association between SES and osteoporosis or HF reported in the literature (not all of which is consistent) ${ }^{9}{ }^{10}$ might be partially explained by unhealthy behaviours such as poor diet, inadequate exercise or harmful occupational environments, which are more frequent in deprived populations. ${ }^{7} 11$

Overall, strong inequalities in temporal trends of $\mathrm{HF}$ incidence have been described between and within countries. ${ }^{12} 13$ Several studies reported increasing trends in HF incidence until the beginning of the last decade (around 2000) and increasing longevity-combined with socioeconomic, demographic and environmental risk factors-have been pointed out as a possible explanation. ${ }^{14} 15$ However, during the past decade (after 2000), stabilisation or a decrease in HF incidence by sex and age groups have also been described in most Western countries and Oceania, ${ }^{16-20}$ and might be the result of prevention strategies, either medical (pharmaceutical treatment for osteoporosis) or non-medical (fall prevention). ${ }^{21} 22$ Even though temporal trends have been widely explored, studies reporting differences in temporal trends by SES are sparse and, to our knowledge, there are no studies reporting how HF incidence rates have changed over time in different regional SES per age group.

To overcome the gap in the literature, we conducted a population-based study in Portugal with the aim to analyse the temporal trends of HF incidence rates during the BJD (2000-2010), by sex and age group, according to municipality in SES regions. 


\section{MATERIAL AND METHODS Study area}

The study area is Continental Portugal-we excluded the two autonomous regions, the archipelagos of Azores and Madeira (5\% of the Portuguese population), from the analysis because there were no available data for these regions. In 2010, there were 1005799 inhabitants distributed heterogeneously throughout 278 municipalities, with a median of 15741 (IQR 7371-39 356) inhabitants per municipality. Portugal is one of the highest aged countries in Europe-27: in 2010 it was the fifth country in the ranking of the highest percentage of persons aged 65 years and over, ${ }^{23}$ with an ageing index of 120 elderly ( $\geq 65$ years of age) per 100 youths ( $\leq 14$ years of age) and $37.7 \%$ of the population over 50 years of age (INE-Statistics Portugal ${ }^{24}$ ).

\section{Data}

We used data from the National Hospital Discharge Register (NHDR), mandatory for all Portuguese public hospitals since 1997. The quality of the NHDR is assessed regularly by internal and external auditors. It is initially carried out in the hospital by internal auditors and later by external auditors from the Central Administration of the National System (ACSS). Internal and external auditors are mandatory medical coders trained by the ACSS. The evaluation of quality is performed systematically in order to determine whether the encoding and resulting grouping Diagnosis Related Groups (DRG) comply with the coding rules, and these are effectively implemented in order to achieve the objectives of the System Patient Classification in DRG. ${ }^{25}$ More details about the NHDR can be found elsewhere; ${ }^{25}$ briefly, each register corresponds to one episode of hospital admission and contains variables such as sex, age, cause of admission (main and up to 19 secondary causes) and diagnosis (main and up to 19 secondary diagnoses) coded according to the International Classification of Diseases, V.9 -Clinical Modification (ICD9-CM), the municipality of patient's residence and the date of admission, among others. For reasons of confidentiality, data were available without information that would allow identification of the patient, such as name, code, address or ID number. During the study period, access to the Portuguese healthcare system (health centres, and hospital consultations and internments) was based on principles of universality, integrality and equity, and were mostly free-of-charge: contributions were based on citizens' socioeconomic conditions ${ }^{26}$ and almost all the patients with HF were treated in public hospitals. Therefore, data of HF hospital admissions from the NHDR can be seen as a proxy of HF incidence in Portugal.

We selected all hospital discharge registers of patients hospitalised from 1 January 2000 to 31 December 2010, aged 50 years and over, with a diagnosis of HF (main, second or third diagnosis) (ICD9-CM codes 820.x) with the main cause being trauma of low/moderate energy (main cause of admission) (ICD9-CM codes E849.0, E849.7 and E880-E888). We excluded readmissions for aftercare (ICD9-CM codes 996.4 and V54.x) and pathological fractures (ICD9-CM codes 170.x and 171.x) if registered in 1 of the 20 . Procedure codes were also taken into consideration to detect and exclude cases with a misclassification readmission on the diagnosis field. Cases with a length of stay less than 5 days and transferred to another hospital without surgery were also excluded (the first hospitalisation could only be for stabilisation of the patient). Counts of HF were stratified by the municipality of patient's residence, admission year, sex and three age groups (50-64, 65-79, 80+ years).

For age-specific incidence rates, we used population counts per municipality from the 2001 Census and from the official estimates for all the other years, ${ }^{24}$ stratified in the same groups of hospital admission data.

Each municipality was classified according to SES, as deprived, medium or affluent; analyses were conducted according to the region's SES. The methods for the classification of municipalities according to SES can be found in detail elsewhere. ${ }^{27}$ In brief, we reduced a set of 30 socioeconomic and demographic variables related to building, households, families and individuals from the 2001 census into four principal components (PCs), based on the Kaiser Criterion (eigenvalues $\geq 1$ ), which retained $75.8 \%$ of the total variability. A varimax rotation was then applied to the four PCs for interpretation and to reduce the dimensionality of the data at a set of uncorrelated variables that account for much of the original data. Later, we conducted a hierarchical cluster analysis based on Ward's method to identify homogenous areas. Municipalities were then aggregated in three clusters of SES that were empirically interpreted as follows: the affluent SES comprises municipalities with younger population, higher educational level, higher percentage of employed individuals, good housing conditions (plumbing, heating, shower and bathroom facilities); compared to the affluent areas, the medium SES comprises municipalities with older populations, higher illiteracy rates, a lower gross domestic product (GDP) per capita and a higher percentage of individuals employed in agricultural, forestry and industry; compared to the affluent and medium areas, the deprived SES comprises municipalities with the highest percentage of elderly, the highest illiteracy rate, the highest rate of people living alone, the lowest level of education, the lowest GDP per capita, the highest percentage of individuals with rural activities, the highest percentage of houses with no running water, no shower and bathroom facilities, and the highest percentage of individuals receiving unemployment benefits. ${ }^{28}$ The population ( $\geq 50$ years of age) significantly differs by SES group ( $p<0.001)$, with the median: 1607 (IQR 1207-2402) in the deprived SES cluster; 3473 inhabitants (IQR 2386-6285) in the medium SES cluster and 7426 inhabitants (IQR 3453-12 706) in the affluent SES cluster.

\section{Statistical analysis}

We computed annual time series of the age-specific incidence rates, expressed as admissions per 100000 persons-year (PY) stratified by sex and regions' SES. The age-specific incidence rates were calculated dividing the total admissions by the total population, per year, sex, SES and age groups 50-64, 65-79 and $80+$ years (the total admissions and population for each year, SES, sex and age group can be seen in online supplementary table S1).

Generalized additive models that incorporate a nonparametric component, s(.), estimated using spline functions (smoothers, useful in revealing possible non-linearities in the effect of the predictors) were fitted to identify shape and turning points where the temporal trend changes significantly in magnitude and/or direction during the study period. Annual (and period) absolute and relative changes over time were quantified between turning points (or between turning points and extremes, in the case of the first and last period) of the time series (more detail can be seen in online supplementary methods 1).

We assumed that the number of HF $\left(\mathrm{NFrat}_{t}\right)$ in a specific year $t$ follows a Poisson distribution with mean $\lambda_{t}=N \operatorname{Nop}_{t} \varrho_{t}$, where NPo $p_{t}$ is the population in year $t$ and $\varrho_{\mathrm{t}}$ is the incidence rate of HF per unit of population in year $t$ (or follows a negative binomial distribution with the scale parameter $\theta$ to account for overdispersion). 
For each sex, age group and regional SES, the parameters of interest were estimated by the following model:

$$
\begin{gathered}
\operatorname{NFrat}_{t} \sim \operatorname{Pois}\left(\lambda_{t}\right) \text { or NFrat }_{t} \sim \operatorname{NegBin}\left(\lambda_{t}, \theta\right) \\
\log \left(\lambda_{t}\right)=\log \left(\operatorname{NPop}_{t}\right)+\log \left(\rho_{t}\right)=\log \left(\operatorname{NPop}_{t}\right)+\beta_{0}+\beta_{1} s\left(\text { Year }^{(t)}\right)
\end{gathered}
$$

The statistical analysis was performed using the packages mgcv and MASS of the statistical software R V.2.14.1 (Project for Statistical Computing).

\section{RESULTS}

There were 98186 admissions, of patients aged 50 years and over, with a diagnosis of HF caused by traumas of low/moderate energy in Continental Portugal between 2000 and 2010. From those, we excluded 585 due to missing data in the municipality of the patient's residence and 696 due to readmissions for after care; our final sample includes 96905 fractures $(77.3 \%$ in women). On average, women are older than men at admission, with a mean age \pm SD of $81.2 \pm 8.5$ vs $78.2 \pm 10.1$ years of age, $\mathrm{p}<0.001$.

Figure 1 shows the temporal trends of age-specific incidence rates (estimated rates for each year, SES, sex and age group are presented in online supplementary table 2 ) and table 1 presents the estimated absolute and relative changes in the trends of agespecific incidence rates per 100000 PY (95\% CI), by regional SES and age groups in women. Only among the group of oldest women are there no differences in the trends of HF due to the region's SES-for this group, we observed a decreasing trend from $2002 / 2004$ to $2006 / 2007$, followed by an increasing trend until the end of the study period. In the other age groups, there are marked SES differences in trends: for younger women (5064 years of age) there are no changes over time, except in the affluent regions, where there are slight decreasing trends (1.9\% HF per year) from 2000 to 2007. For women aged 6579 years: in the affluent regions, there is a continuously decreasing trend in the overall period $(1.7 \% \mathrm{HF}$ per year) while for the medium regions, there is an abrupt decreasing trend $(4.7 \% \mathrm{HF}$ per year) for a short period (2004-2007) followed by an increasing trend (3.4\% HF per year). On the other hand, in the deprived regions, only an increasing trend was observed (20062010), with an increment of 3.3\% HF per year.

Similar analyses were conducted for men and the results are presented in figure 2 and table 2. In general, men present fewer differences in the trends compared to women. In younger men (50-64 years of age), no significant changes in the trends are observed. In the 65-79-year age group, the affluent regions present a decrease of 1.8\% HF per year between 2001 and 2007; on the contrary, the deprived regions have an annual increase of $4.4 \% \mathrm{HF}$ per year, from 2004 to 2010. For the oldest men ( $\geq 80$ years of age), a similar pattern was observed in affluent and deprived regions with a stable period followed by an increasing tendency (2006-2010), significant only in affluent regions (an increment of $1.6 \% \mathrm{HF}$ per year). Contrary to the other regional SES, the medium regions present a decreasing trend from 2003 to 2007 (2.3\% HF per year).

\section{DISCUSSION}

In this study, we aimed to analyse the temporal trends of agespecific incidence rates of $\mathrm{HP}$, by sex, age groups and regional SES in Portugal, during the BJD (2000-2010). We found socioeconomic, age and sex inequalities in temporal trends. Inequalities are avoidable differences and usually linked to unequal opportunities of access to prevention or treatment. ${ }^{29}$
For the oldest women ( $\geq 80$ years of age) the SES inequalities in the trends seem to vanish, and this might be because social inequalities can diminish with age. ${ }^{30}$ The most disadvantaged survivors are in general very healthy since the less healthy will have less probability of reaching older age. ${ }^{30}$ Only women aged 65-79 years in the affluent regions, have a continuously decreasing trend during all the study period. The strongest inequalities were observed in women between 65 and 79 years of age and increased during the BJD: while $\mathrm{HF}$ incidence persistently decreases in the affluent regions, the decrease in the medium regions was only for a short period of 3 years and, in the deprived regions, there was no decreasing period; besides, there are increasing trends starting first in 2006 in the deprived regions and later, in 2007, in the medium regions. Our results seem to show that HF reduction from 2000 to 2010 have mainly reached a restricted group: women aged 65-79 years in the affluent regions, the preferential target population for medication and treatment against osteoporosis and $\mathrm{HF}^{31}{ }^{32}$ while sustainable reduction seems to have failed for all the other men and women. All stable or increasing trends in 2000 (for all age groups and regional SES among women) turned to accentuate decreasing trends around 2002/2003, although such turning points were rarely observed for men. Abrupt changes in epidemiological trends are usually compatible with interventions, or other events affecting population health, rather than with the natural development of a disease. Apparently, in the beginning of BJD, there were positive efforts to reduce HF. A National Program Against Rheumatic Disease (NPARD) was implemented by the Ministry of Health as a contribution to the BJD initiative. The programme put these diseases in the national political agenda, by pooling efforts of services and levels of healthcare provision, and investment on primary, secondary and tertiary prevention. The NPARD had the final goal of inverting the increasing trend of the problem. ${ }^{33}$ Also, a previous study identified a sales increase, around 2003, of first-line medication against osteoporosis, mainly bisphosphonates, coincident with decreasing trends of HF incidence among women in the 6579 -year age group. ${ }^{34}$ However, our results show that in most of the analysed groups the decreasing trends last for short periods and new turning points with increasing trends started in 2006/ 2007, continuing until 2010 and nullifying the positive effect of the decreasing periods-at the end of the BJD, the age-specific incidence rates in women (except those from affluent regions, with ages 65-79 years) are in the same magnitude, or even higher, than in the beginning of the BJD and the SES inequalities deepened. Some reasons might explain the abrupt turning points to increasing trends after 2006/2007. The well-being of the population worsened, especially among the elderly ( $\geq 65$ years of age) due to a revision (lowering) of the pensions in $2007 . .^{35}$ Since 2007, the economic scenario has worsened as Portugal faced severe consequences of the global economic/ financial crisis. ${ }^{36}$ Experience and evidence from past recessions show a negative impact of austerity policies on heath inequality. ${ }^{37}$ Recession periods can impact people's lifestyles in several ways, which in turn might affect long-term HF rates, although we would expect that such an impact would reflect smooth changes in the trends of HF. Abrupt changes, such as what we observed, are more likely to be due to abrupt changes in society with impacts on the population. The abrupt turn from decreased to increased HF rates could be due to changes in the guidelines for prescriptions of antiosteoporosis medication, for instance. An analysis of medical prescriptions in the study period could have been elucidative. Unfortunately, such data were not available. However, there is no reason to believe that 
Affluent

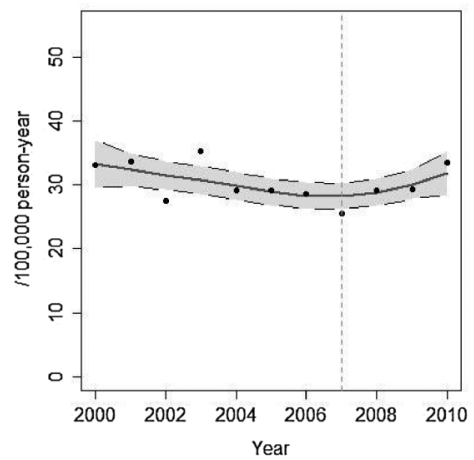

Women 65-79

Affluent

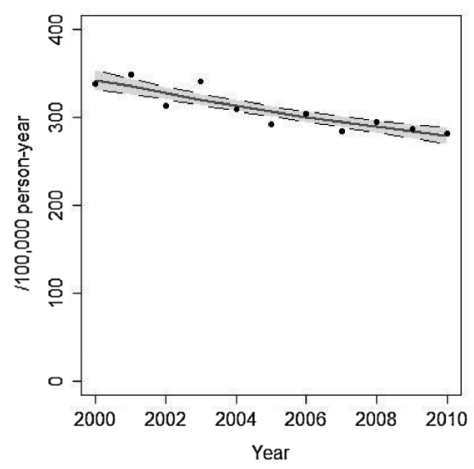

Women 80+

Affluent

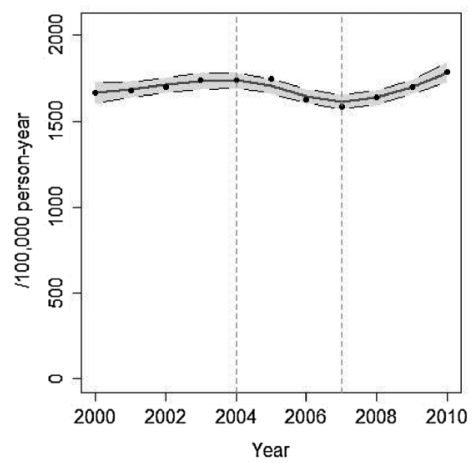

Medium

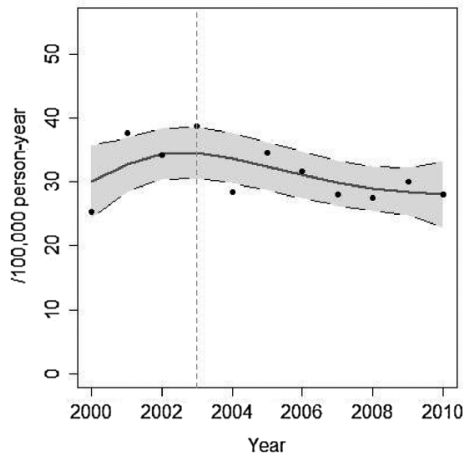

Medium

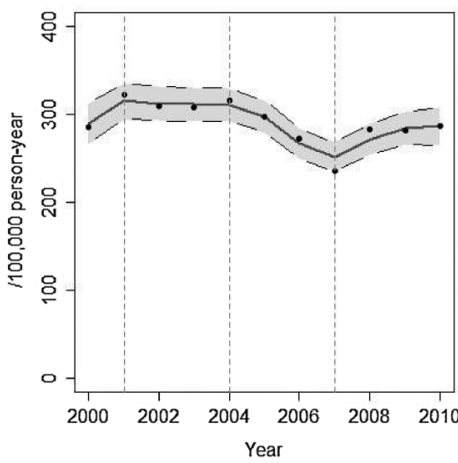

Medium

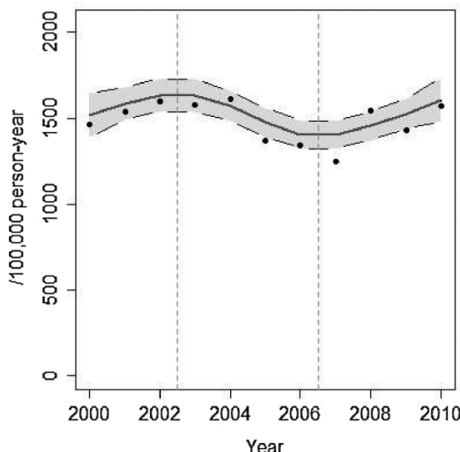

Deprived

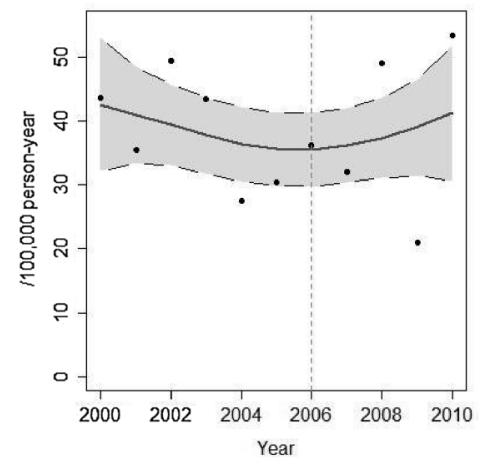

Deprived

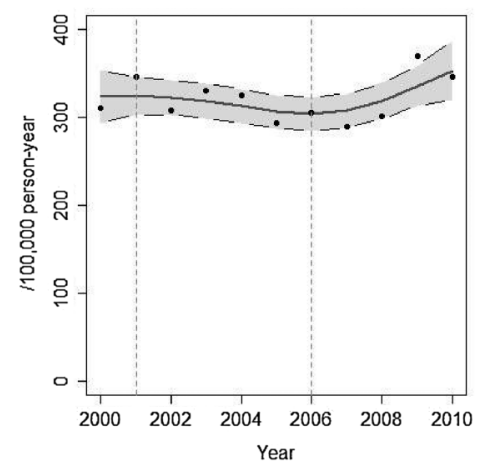

Deprived

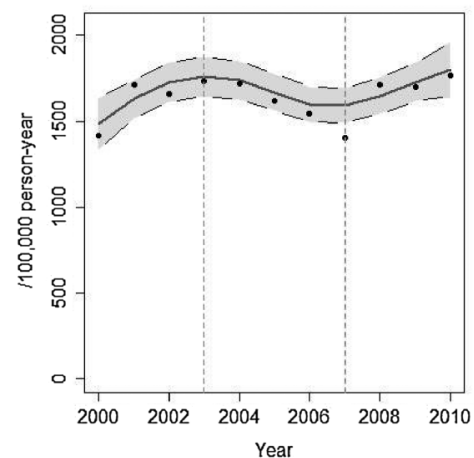

Figure 1 Estimated temporal trends (2000-2010) of age-specific incidence rates of hip fracture, per 100000 persons-year (95\% Cl) by socioeconomic status regions and age groups in women.

during the study period physicians reduced the prescriptions, since there was neither public disinvestment in health ${ }^{36}$ nor changes in the Portuguese guidelines for osteoporotic treatment. Reduction in antiosteoporosis medication prescriptions could also be due to new evidence about the adverse effects of longterm use of bisphosphonates. ${ }^{38} 39$ Nevertheless, this hypothesis does not seem to be a reasonable explanation for the observed differences in trends of HF because a reduction in prescriptions, whatever the reason, would affect all the SES-age-sex groups equally. Loss of adherence to antiosteoporosis treatment could explain the increasing trends after 2006/2007. In the USA, studies evaluating the adherence to osteoporosis medications pointed out the main reasons for abandonment: drug-related side effects, having multiple comorbid conditions and higher cost. $^{40} 41$ In Portugal, some patients reported difficulties in accessing medicaments due to economic constraints, drugs for chronic conditions being among those that patients most often failed to acquire. ${ }^{36}$ The percentage of government participation in the costs of medications in Portugal varies according to the disease to be treated/controlled. Antiosteoporotic medicaments (including generic) are only partially supported by the government; therefore, all patients need to contribute a certain percentage (which varies according to the medication). For older patients (who tend to be polymedicated) and for the most deprived patients, any cost, however small, might be a heavy burden in their budget. The more vulnerable segment of the 


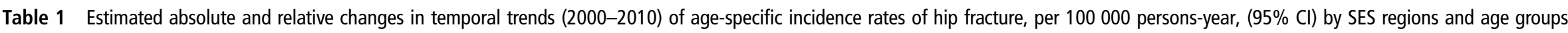
in women

\begin{tabular}{|c|c|c|c|c|c|c|c|}
\hline AgGr & SES & Period & AIR & AAC & PAC & ARC & PRC \\
\hline \multirow[t]{6}{*}{ AgGr50-64 } & Affluent & $2000-2007^{*}$ & $33.2(29.5$ to 36.9$)$ & $-0.6(-1.2$ to -0.1$)$ & $-5.1(-9.2$ to -0.9$)$ & $-1.9(-3.2$ to -0.4$)$ & $-15.3(-25.6$ to -3.2$)$ \\
\hline & & 2007-2010 & 28.2 (26.2 to 30.2$)$ & $0.9(0.0$ to 1.8$)$ & $3.7(-0.1$ to 7.4$)$ & $3.2(-0.1$ to 7.0$)$ & $12.8(-0.2$ to 28$)$ \\
\hline & Medium & $2000-2003$ & 30.1 (24.4 to 35.7 ) & $1.2(-0.7$ to 2.8$)$ & $4.8(-2.9$ to 11.1$)$ & $4(-2.1$ to 11.1$)$ & $15.9(-8.4$ to 44.5$)$ \\
\hline & & $2003-2010$ & 34.5 (30.6 to 38.5 ) & $-0.8(-1.6$ to 0.0$)$ & $-6.4(-13.1$ to 0.1$)$ & $-2.3(-4.6$ to 0$)$ & $-18.5(-36.4$ to 0.2$)$ \\
\hline & Deprived & 2000-2007 & 42.6 (32.1 to 53.0 ) & $-0.9(-2.6$ to 0.8$)$ & $-6.6(-18$ to 5.5$)$ & $-2.2(-5.1$ to 2.5$)$ & $-15.5(-35.8$ to 17.3$)$ \\
\hline & & 2007-2010 & 36.1 (30.3 to 42.0$)$ & $1.1(-1.4$ to 3.6$)$ & $5.7(-6.8$ to 18.1$)$ & $3.2(-3.5$ to 10.9$)$ & $15.8(-17.4$ to 54.6$)$ \\
\hline \multirow[t]{8}{*}{ AgGr65-79 } & Affluent & $2000-2010^{*}$ & 342.6 (331.5 to 353.7 ) & $-5.8(-7.1$ to -4.5$)$ & $-63.3(-78$ to -49.3$)$ & $-1.7(-2.0$ to -1.3$)$ & $-18.5(-22.2$ to -14.8$)$ \\
\hline & Medium & $2000-2001$ & 289.0 (266.2 to 311.8$)$ & $13.4(-1.2$ to 28.2$)$ & $26.7(-2.5$ to 56.5$)$ & $4.6(-0.4$ to 10.6$)$ & $9.2(-0.8$ to 21.1$)$ \\
\hline & & 2001-2004 & 315.3 (295.1 to 335.5$)$ & $-1.1(-7.8$ to 5.9$)$ & $-4.5(-31.1$ to 23.7$)$ & $-0.4(-2.4$ to 2.0$)$ & $-1.4(-9.4$ to 7.8$)$ \\
\hline & & $2004-2007^{*}$ & 310.8 (292.1 to 329.5$)$ & $-14.7(-21.1$ to -8.6$)$ & $-58.8(-84.2$ to -34.5$)$ & $-4.7(-6.5$ to -2.9$)$ & $-18.9(-26.1$ to -11.7$)$ \\
\hline & & $2007-2010^{*}$ & $251.7(235.2$ to 268.1$)$ & 8.5 (2.2 to 15.2$)$ & 34.1 (8.9 to 61$)$ & 3.4 (0.9 to 6.3$)$ & 13.5 (3.5 to 25.2$)$ \\
\hline & Deprived & 2000-2001 & 323.3 (293.1 to 353.6 ) & $0.5(-17.3$ to 19.4$)$ & $0.9(-34.5$ to 38.8$)$ & 0.1 (-4.9 to 6.5$)$ & 0.3 (-9.8 to 13$)$ \\
\hline & & 2001-2006 & 324.4 (303.2 to 345.6$)$ & $-3.4(-7.9$ to 0.7$)$ & $-20.6(-47.5$ to 4.1$)$ & $-1.1(-2.4$ to 0.2$)$ & $-6.3(-14.1$ to 1.3$)$ \\
\hline & & $2006-2010^{*}$ & 303.8 (284.7 to 322.9$)$ & $10.0(2.3$ to 17.3$)$ & 49.8 (11.4 to 86.4$)$ & $3.3(0.7$ to 6.0$)$ & 16.4 (3.6 to 29.9 ) \\
\hline \multirow[t]{9}{*}{ AgGr80mais } & Affluent & $2000-2004$ & 1663.2 (1599.7 to 1726.8$)$ & $14.5(-0.6$ to 29.9$)$ & $72.5(-3.1$ to 149.5$)$ & $0.9(0.0$ to 1.8$)$ & $4.4(-0.2$ to 9.2$)$ \\
\hline & & $2004-2007^{*}$ & 1737.0 (1693.1 to 1780.9$)$ & $-31.5(-45.9$ to -16.5$)$ & $-126(-183.5$ to -66.1$)$ & $-1.8(-2.6$ to -1.0$)$ & $-7.2(-10.4$ to -3.8$)$ \\
\hline & & $2007-2010^{*}$ & 1611.4 (1571.3 to 1651.6$)$ & 43.1 (26.2 to 59.7$)$ & 172.2 (104.8 to 238.7$)$ & 2.7 (1.6 to 3.8$)$ & $10.7(6.4$ to 15.1$)$ \\
\hline & Medium & $2001-2002$ & 1514.8 (1385.9 to 1643.8 ) & $34.4(-10.7$ to 76.9$)$ & 120.6 ( -37.4 to 269.2$)$ & $2.3(-0.7$ to 5.4$)$ & $7.9(-2.3$ to 18.9$)$ \\
\hline & & $2002-2006^{*}$ & 1634.6 (1539.9 to 1729.3$)$ & $-46.7(-70.4$ to -20.9$)$ & $-233.6(-352.2$ to -104.7$)$ & $-2.9(-4.2$ to -1.3$)$ & $-14.3(-20.9$ to -6.7$)$ \\
\hline & & $2006-2010^{*}$ & 1403.8 (1324.1 to 1483.4$)$ & 45.1 (13.1 to 81.8$)$ & 203.2 (58.8 to 368.2 ) & $3.2(0.9$ to 6.1$)$ & 14.5 (4 to 27.2$)$ \\
\hline & Deprived & $2000-2003^{*}$ & $1486.2(1337.4$ to 1635.0$)$ & $68.9(22.2$ to 115$)$ & 275.8 (88.9 to 460.0$)$ & $4.7(1.4$ to 8.5$)$ & 18.6 (5.6 to 33.8$)$ \\
\hline & & $2003-2007^{*}$ & 1759.8 (1646.0 to 1873.7$)$ & $-34.1(-62.0$ to -3.6$)$ & $-170.6(-309.9$ to -18.0$)$ & $-1.9(-3.4$ to -0.2$)$ & $-9.7(-16.8$ to -1.1$)$ \\
\hline & & $2007-2010^{*}$ & 1591.8 (1490.0 to 1693.7$)$ & 52.1 (9.1 to 98.6$)$ & 208.4 (36.6 to 394.4 ) & $3.3(0.6$ to 6.4$)$ & $13.1(2.2$ to 25.4$)$ \\
\hline
\end{tabular}

AAC, annual absolute change, per 100000 persons-year; AgGr, age group; AIR, age-specific incidence rates, per 100000 persons-year, in the beginning of the period; ARC, annual relative change (\%); PAC, period absolute change, per 100000 persons-year; PRC, period relative change (\%); SES, regional socioeconomic status.

${ }^{*} \mathrm{p}<0.05$ for temporal trend (significant change). 
Affluent

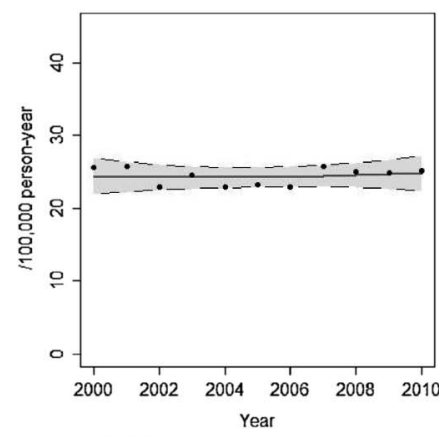

Men 65-79

Affluent

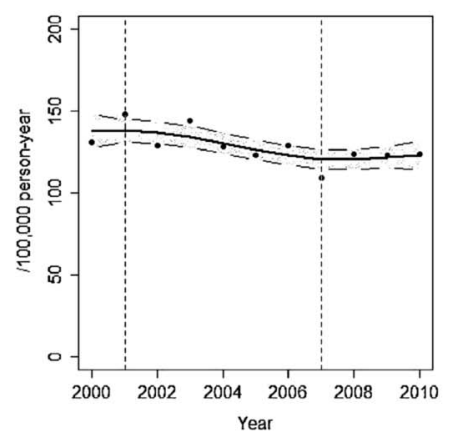

Men 80+

Affluent

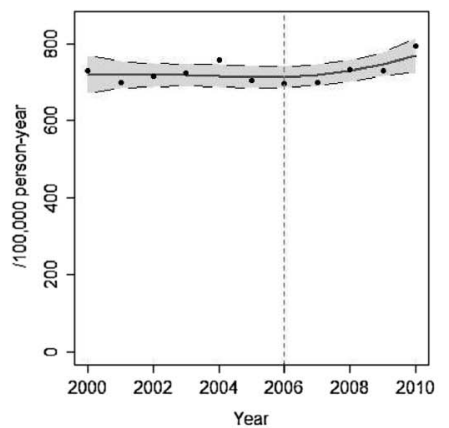

Medium

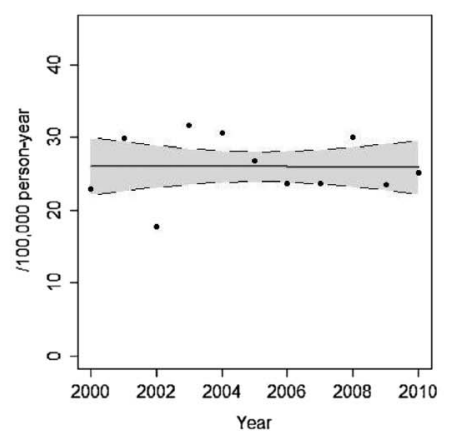

Medium

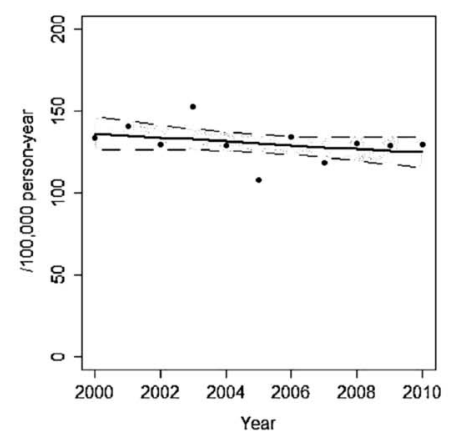

Medium

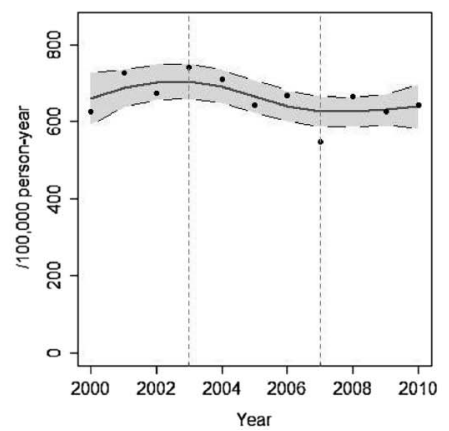

Deprived

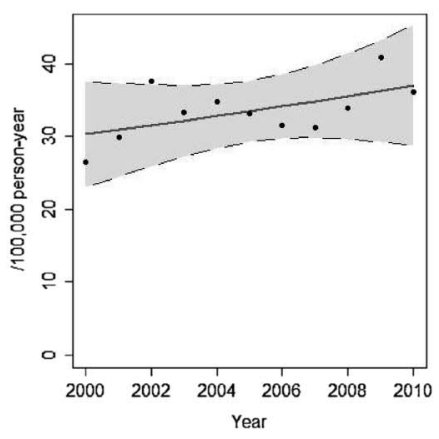

Deprived

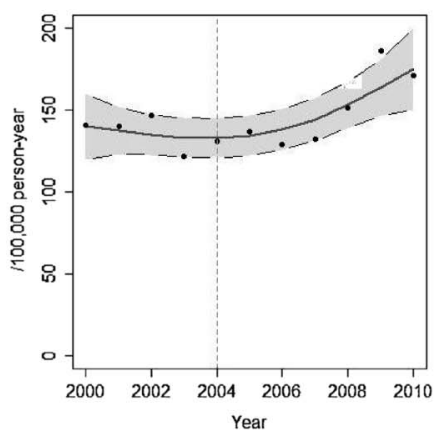

Deprived

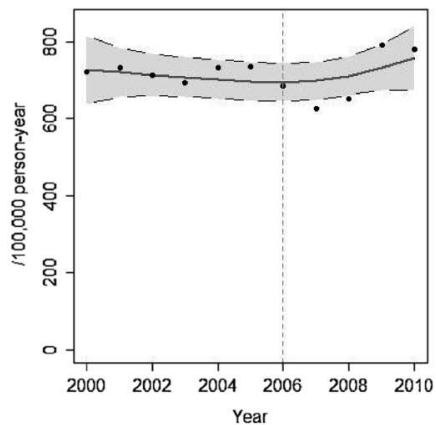

Figure 2 Estimated temporal trends (2000-2010) of age-specific incidence rates of hip fracture, per 100000 persons-year (95\% Cl) by socioeconomic status regions and age groups in men.

population is usually more adversely affected by poverty, job insecurity, unemployment, and privatisation of goods and services. Vulnerability results from an interaction between developmental problems, personal incapacities, disadvantaged social status, the inadequacy of interpersonal networks and support, degraded neighbourhoods and environments. ${ }^{42}$ In times of crisis, there is expected to be a deterioration of healthcare seeking, various effects on providers, and a disease burden that will especially affect the most deprived and the elderly population. ${ }^{36}$ The loss of adherence due to economic constraints is a plausible explanation for the trend patterns observed, since the elderly and the more deprived population were the most affected by the economic crisis and it is in these groups that increasing trends were observed after 2007. Moreover, the absence of a lag time between economic crisis and the increasing turning points corroborates the hypothesis of loss of adherence of antiosteoporotic medication. ${ }^{34}$ Most of the studies on the health effects of the economic crisis have analysed aggregate data focusing on population averages, and may have hidden the existent inequalities between SES. Our online supplementary material is an example of this; we present the results of an analysis for the whole period in each age and sex group, but without SES stratification, and we lose the capacity to identify differences among groups. We observed roughly the same pattern in all sex and age groups (two turning points), except for men aged 50-64 years, and at different scales (see online supplementary methods 2, figures S1, S2, tables S3 and S4). It seems that analysing aggregated data (as most of the published studies do) may hide SES inequalities. For example, in our study, when we analysed HF without stratifying by SES groups, we did not identify the decreased trend of HF in women aged 65-79 years in affluent areas. In addition, inequalities tend to rise during recessions since those of affluent SES are better at adapting to new and changing economic circumstances than 
Table 2 Estimated absolute and relative changes in temporal trends (2000-2010) of age-specific incidence rates of hip fracture, per 100000 persons-year, $(95 \% \mathrm{Cl})$ by SES regions and age groups in men

\begin{tabular}{|c|c|c|c|c|c|c|c|}
\hline $\mathrm{AgGr}$ & SES & Period & AIR & AAC & PAC & ARC & PRC \\
\hline \multirow[t]{3}{*}{ AgGr50-64 } & Affluent & $2000-2010$ & 24.4 (21.9 to 26.9$)$ & $0.0(-0.3$ to 0.3$)$ & $0.5(-3.3$ to 3.8$)$ & $0.2(-1.1$ to 1.5$)$ & $2.2(-12.5$ to 16.4$)$ \\
\hline & Medium & 2000-2010 & 26.0 (22.1 to 30.0$)$ & $0.0(-0.5$ to 0.5$)$ & $-0.1(-5.2$ to 5.1$)$ & 0.0 (-1.7 to 2$)$ & $-0.5(-18.8$ to 21.5$)$ \\
\hline & Deprived & 2000-2010 & 30.3 (23.0 to 37.5 ) & $0.6(-0.3$ to 1.6$)$ & $6.6(-3.7$ to 18.1$)$ & $2.0(-1$ to 6.4$)$ & 21.6 (-10.7 to 70.1$)$ \\
\hline \multirow[t]{6}{*}{ AgGr65-79 } & Affluent & $2000-2001$ & 137.6 (127.3 to 147.8$)$ & 0.0 (-6.1 to 6.5$)$ & $0.0(-12.2$ to 13$)$ & $0.0(-4.0$ to 5.1$)$ & $0.0(-8.1$ to 10.2$)$ \\
\hline & & $2001-2007^{*}$ & 137.9 (130.8 to 145.1$)$ & $-2.5(-3.8$ to -1.1$)$ & $-17.3(-26.3$ to -7.4$)$ & $-1.8(-2.6$ to -0.8$)$ & $-12.6(-18.3$ to -5.6$)$ \\
\hline & & $2007-2010$ & 120.5 (114.6 to 126.4$)$ & 0.6 ( -2.0 to 3.3$)$ & $2.4(-8.1$ to 13.2$)$ & 0.5 (-1.6 to 2.8$)$ & $2.0(-6.5$ to 11.3$)$ \\
\hline & Medium & $2000-2010$ & 136.4 (125.9 to 146.8$)$ & $-1.1(-2.4$ to 0.2$)$ & -11.8 ( -26.3 to 2.3$)$ & $-0.8(-1.7$ to 0.2$)$ & $-8.6(-18.2$ to 1.8$)$ \\
\hline & Deprived & $2000-2004$ & 139.8 (119.8 to 159.8$)$ & $-1.5(-6.2$ to 3.2$)$ & $-7.4(-31.1$ to 16.2$)$ & $-1.1(-4$ to 2.5$)$ & $-5.3(-19.9$ to 12.7$)$ \\
\hline & & 2004-2010* & 132.8 (120.8 to 144.9$)$ & 5.9 (1.9 to 10.2$)$ & 41.6 (13.2 to 71.4$)$ & 4.4 (1.4 to 7.9$)$ & 31.1 (9.9 to 55.5$)$ \\
\hline \multirow[t]{7}{*}{ AgGr80mais } & Affluent & 2000-2006 & 719.7 (670.8 to 768.5$)$ & $-0.8(-9.1$ to 7.6$)$ & $-5.6(-63.6$ to 53.2$)$ & $-0.1(-1.2$ to 1.1$)$ & $-0.8(-8.3$ to 7.9$)$ \\
\hline & & $2006-2010^{*}$ & 713.1 (685.4 to 740.8 ) & $11.1(0.5$ to 20.8$)$ & 55.3 (2.7 to 103.9$)$ & $1.6(0.1$ to 3.0$)$ & $7.8(0.4$ to 14.9$)$ \\
\hline & Medium & 2000-2003 & 660.3 (592.3 to 728.4 ) & 11.5 (-8.8 to 31.4$)$ & $45.8(-35.0$ to 125.6$)$ & $1.7(-1.3$ to 5.2$)$ & $7.0(-5.1$ to 20.7$)$ \\
\hline & & $2003-2007^{*}$ & 706.0 (661.0 to 751.0$)$ & $-16.2(-28.3$ to -4.6$)$ & $-81.0(-141.4$ to -22.8$)$ & $-2.3(-3.8$ to -0.7$)$ & $-11.5(-19.1$ to -3.4$)$ \\
\hline & & $2007-2010$ & 625.1 (586.0 to 664.2 ) & 3.5 (-13.0 to 20.0 ) & 13.8 (-52.0 to 80.2$)$ & $0.5(-2.0$ to 3.3$)$ & $2.2(-8.0$ to 13.3$)$ \\
\hline & Deprived & $2000-2006$ & 726.5 (638.8 to 814.1$)$ & $-4.8(-19.1$ to 9.9$)$ & $-33.3(-133.7$ to 69.1$)$ & $-0.7(-2.4$ to 1.6$)$ & $-4.6(-16.5$ to 10.9$)$ \\
\hline & & $2006-2010$ & 694.3 (644.9 to 743.6 ) & $12.2(-6.0$ to 31.0$)$ & $60.8(-29.8$ to 154.9$)$ & $1.7(-0.8$ to 4.6$)$ & $8.7(-4.2$ to 23.1$)$ \\
\hline
\end{tabular}

AAC, annual absolute change, per 100000 persons-year; AgGr, age group; AIR, age-specific incidence rates, per 100000 persons-year, in the beginning of the period; ARC, annual relative change (\%); PAC, period absolute change, per 100000 persons-year; PRC, period relative change (\%); SES, regional socioeconomic status.

${ }^{*} \mathrm{p}<0.05$ for temporal trend (significant change).

those of deprived SES. Among men there are also SES inequalities in the trends, although not so evident as among women. ${ }^{43}$

We found age inequalities in the temporal trends of HF incidence, with the oldest women in a disadvantageous position. Only women over 80 years of age show increasing trends in all the SES regions after 2006/2007, reinforcing our hypothesis that the economic crisis in Portugal, which had a high impact on the elderly regardless of their SES, may have contributed to the observed trends. The elderly are usually polymedicated due to several comorbidities and the antiosteoporosis medication is among the first abandoned medications ${ }^{40} 44$ in the face of economic constraints. Furthermore, only for women aged $\geq 80$ years from deprived regions was there an increasing trend in the beginning of the BJD, and the later observed decreasing periods were shorter in this age group. This might reflect age inequalities in the priorities of intervention actions, targeting younger women while the oldest are neglected. ${ }^{42}$ In general, temporal trends are more stable in the youngest group of those aged 50-64 years. Statistically, there is only one significant reduction period among women aged 50-64 years (0.6 HF per 100000 per person-year in affluent SES). However, the magnitude of this reduction is too small and might arguably be clinically irrelevant.

There are sex inequalities evident in the trends: during the study period women in all age groups and SES regions (except those aged $<80$ years in deprived regions) presented periods of significant decreasing trends in the age-specific incidence rates. Among men, a reduction was observed only in two groups: ages $65-79$ years in affluent regions, and ages $\geq 80$ years in the medium regions. In general, trends among men are stable and this might be a consequence of the lack of attention to men's bone health. Frequently, osteoporosis and $\mathrm{HF}$ are seen as a woman's problem and less attention is given to men in bothprevention programmes and drug therapy. In men, drug therapy has never reached the level that it has in women; ${ }^{45}$ although, with their increasing longevity, more men will be at risk for osteoporosis and $\mathrm{HF}^{43}$ and interventions must be more inclusive of men.
A limitation of our study is the non-existence of individual data for the patients' SES characterisation because the individual's socioeconomic data is not routinely collected. Therefore, any conclusions need to be treated with caution, even though the SES of the place of residence is frequently and adequately used as a proxy of individual SES. ${ }^{10}{ }^{46}$ Furthermore, the measures of municipality SES are helpful to recognise geographical areas at high-risk of HF in order to better direct public health intervention programmes. Recurrent fractures could not be identified because of confidentiality issues (the patient unique identifier was not available), and this could be seen as a limitation since the risk of a new fragility fracture increases after the primary HF. However, our study focused on incidence rates of HF (number of HF in a population at risk) rather than cumulative incidence (number of persons with an HF in a population at risk) and, therefore, such a limitation does not bias our results.

Our study has the advantage of using population based on repeated cross-sectional data from a nationwide register and stratifies the HF incidence per SES regions, sex and age groups, allowing the identification of different effects on trends and clarifying hidden inequalities in the trends. The use of a multidimensional SES that encapsulates a variety of domains, related to building, households, families and individual characteristics, and determined by an approach that allows reduction of the covariates to a set of uncorrelated variables that explain much of the original data, can be seen as an advantage. Despite the focus that the BJD brought to studies on HF, little attention has been given to the inequalities associated with this disorder and, to our knowledge, this is the first study analysing the temporal trends of HF incidence by sex and age group for different SES regions.

Our results can give insights on the real burden of HF and help with better planning of health policies. Understanding positive and negative aspects of the initiatives triggered by BJD may help to potentiate better results in the ongoing global alliance for musculoskeletal health. In general, the abrupt decreasing turning points found in our study seem to be plausibly 
explained by an intervention, likely based on increased treatment (eg, antiosteoporosis medication) and prevention programmes observed since the beginning of the BJD, with an impact on the incidence rates in all ages and SES groups among women. Coincident with the beginning of the economic crisis in Portugal, the most deprived and oldest groups returned to increased trends, reaching similar or even higher rates in 2010 than those observed in 2000.

\section{CONCLUSIONS}

There are socioeconomic, age and sex inequalities in temporal trends of age-specific incidence rates in Portugal during the BJD. The inequalities in trends are especially marked in women between 65 and 79 years of age: only those living in affluent areas have a continuously decreasing trend while the medium and deprived regions have increasing trends after 2007. The SES inequalities observed in women aged $<80$ years disappear in the oldest age group ( $\geq 80$ years). It seems that HF prevention during the BJD in Portugal has reached mainly women (6579 years of age) in the affluent areas while it has failed for all the other age groups. Clear sex inequalities were observed: only two groups of SES/age in men presented a decreasing period, as opposed to the majority of SES/age groups in women. Overall, the trends among men are stable, which could reflect the lack of attention given to bone health among men.

\section{What is already known on this subject}

- An inverse association between socioeconomic status and osteoporosis or hip fracture (HF) (not all consistent) has been generally reported in cross-sectional studies, probably due to unhealthy behaviours such as poor diet, inadequate exercise or harmful occupational environments, which are more frequent in deprived populations.

\section{What this study adds}

- Even though temporal trends have been widely explored, studies reporting differences in temporal trends by socioeconomic status (SES) are sparse.

- Our results are surprising and may contribute to the discussion and reflection of current interventions to reduce HF.

- Incidence rates of HF have changed over time in different regional SES per age group and sex.

- Women aged 65-79 years, who are frequently the preferential target population for osteoporosis and $\mathrm{HF}$ prevention, show the strongest SES inequalities: only those from affluent regions have a continuous decreasing trend, whereas those from deprived regions have had no decreasing and, on the contrary, have had an increasing trend since 2006/2007.

- Surprisingly, the SES inequalities vanish among the oldest women ( $\geq 80$ years) -all of them, regardless of the regional SES, show increasing trends after 2006/2007 similar to those occurring in both sexes and all ages from deprived regions (although not all are significant).

- For men, overall temporal trends are stable, which could reflect the lack of attention given to bone health in men.
Despite some successful periods of decreasing trends, at the end of the BJD, the incidence rates returned to the values of 2000 , or were even higher, in almost all age groups, in men and women.

\section{REFERENCES}

1 Wilkins CH, Birge SJ. Prevention of osteoporotic fractures in the elderly. Am J Med 2005; 118:1190-5.

2 Hernlund E, Svedbom A, Ivergard M, et al. Osteoporosis in the European Union: medical management, epidemiology and economic burden. A report prepared in collaboration with the International Osteoporosis Foundation (IOF) and the European Federation of Pharmaceutical Industry Associations (EFPIA). Arch Osteoporos 2013;8:136

3 BJD. The bone and joint decade. Global alliance for musculoskeletal heath. 2015 cited 20015 06-11-2015. http://bjdonline.org/

4 Woolf AD. The bone and joint decade 2000-2010. Ann Rheum Dis 2000;59:81-2.

5 Mackenbach JP. Health inequalities: Europe in profile. UK: WHO European Region, 2006.

6 Woolf AD. Bone and Joint Decade report: moving together beyond the decade. Preface. Best Pract Res Clin Rheumatol 2012;26:167-8.

7 Adler NE, Newman K. Socioeconomic disparities in health: pathways and policies. Health Aff (Millwood) 2002;21:60-76.

8 Wilkinson R, Marmot M. Social determinants of health: the solid facts. Copenhagen, Denmark: World Health Organization Europe, 2003.

9 Brennan SL, Pasco JA, Urquhart DM, et al. Association between socioeconomic status and bone mineral density in adults: a systematic review. Osteoporos Int 2011;22:517-27

10 Brennan SL, Pasco JA, Urquhart DM, et al. The association between socioeconomic status and osteoporotic fracture in population-based adults: a systematic review. Osteoporos Int 2009;20:1487-97.

11 Pampel FC, Krueger PM, Denney JT. Socioeconomic disparities in health behaviors. Annu Rev Sociol 2010;36:349-70.

12 Cheng SY, Levy AR, Lefaivre KA, et al. Geographic trends in incidence of hip fractures: a comprehensive literature review. Osteoporos Int 2011;22:2575-86.

13 de Pina MF, Alves SM, Barbosa M, et al. Hip fractures cluster in space: an epidemiological analysis in Portugal. Osteoporos Int 2008;19:1797-804.

14 Hagino $\mathrm{H}$, Katagiri $\mathrm{H}$, Okano $\mathrm{T}$, et al. Increasing incidence of hip fracture in Tottori Prefecture, Japan: trend from 1986 to 2001. Osteoporos Int 2005;16:1963-8.

15 Kannus P, Niemi S, Parkkari J, et al. Hip fractures in Finland between 1970 and 1997 and predictions for the future. Lancet 1999;353:802-5.

16 Ballane G, Cauley JA, Luckey MM, et al. Secular trends in hip fractures worldwide: opposing trends East versus West. J Bone Miner Res 2014;29:1745-55.

17 Kannus P, Niemi S, Parkkari J, et al. Nationwide decline in incidence of hip fracture. J Bone Miner Res 2006;21:1836-8.

18 Dimai HP, Svedbom A, Fahrleitner-Pammer A, et al. Epidemiology of hip fractures in Austria: evidence for a change in the secular trend. Osteoporos Int 2011;22:685-92.

19 Briot K, Maravic M, Roux C. Changes in number and incidence of hip fractures over 12 years in France. Bone 2015;81:131-7.

20 Piscitelli $\mathrm{P}$, Feola $\mathrm{M}$, Rao C, et al. Ten years of hip fractures in Italy: for the first time a decreasing trend in elderly women. World J Orthop 2014;5:386-91.

21 Melton LJ III, Kanis JA, Johnell O. Potential impact of osteoporosis treatment on hip fracture trends. J Bone Miner Res 2005;20:895-7.

22 Feder G, Cryer C, Donovan S, et al. Guidelines for the prevention of falls in people over 65. The Guidelines' Development Group. BMJ 2000;321:1007-11.

23 Population Ageing in the United Kingdom, its Constituent Countries and the European Union. http://www.ons.gov.uk/ons/dcp171776_258607.pdf2012 cited 2014 14-12-2104.

24 INE. Instituto Nacional de Estatística; cited 2012. http://www.ine.pt

25 ACSS. Auditoria da codificação clínica Clinical codification Audits. 2011. http:/l portalcodgdh.min-saude.pt/index.php/Auditoria_da_codificação_cl\%C3\%ADnica

26 Barros PP, Machado SR, Simoes Jde A. Portugal. Health system review. Health Syst Transit 2011;13:1-156.

27 Alves SM, Castiglione D, Oliveira CM, et al. Age-period-cohort effects in the incidence of hip fractures: political and economic events are coincident with changes in risk. Osteoporos Int 2014;25:711-20.

28 Oliveira CM, Economou T, Bailey T, et al. The interactions between municipal socioeconomic status and age on hip fracture risk. Osteoporos Int 2015;26:489-98.

29 Braveman PA, Kumanyika S, Fielding J, et al. Health disparities and health equity: the issue is justice. Am J Public Health 2011;101(Suppl 1):S149-55.

30 Jefferys M. Social inequalities in health-do they diminish with age? Am J Public Health 1996:86:474-5.

31 NCGC. Osteoporosis: fragility fracture risk. Osteoporosis: assessing the risk of fragility fracture. London: NCGC, National Clincal Guideline Centre, 2012.

32 Etxebarria-Foronda I, Arrospide A, Soto-Gordoa M, et al. Regional variability in changes in the incidence of hip fracture in the Spanish population (2000-2012). Osteoporos Int 2015;26:1491-7. 
33 Lucas R, Monjardino M. 0 estado da Reumatologia em Portugal. 2010.

34 Lucas R, Monjardino M. O estado da Reumatologia em Portugal. Observatório Nacional das Doenças Reumáticas. Programa Nacional Contra as Doenças reumáticas. 2010.

35 Murteira MC. A reforma de 2007 do sistema público de pensões em Portugaluma análise crítica das escolhas normativas implícitas. Faculdade de Economia da Universidade de Coimbra, 2008.

36 Sakellarides C, Castelo-Branco L, Barbosa P, et al. The impact of the financial crisis on the health system and health in Portugal. Copenhagen, Denmark: World Health Organization, 2014.

37 Bacigalupe A, Escolar-Pujolar A. The impact of economic crises on social inequalities in health: what do we know so far? Int J Equity Health 2014;13:52.

38 Kennel KA, Drake MT. Adverse effects of bisphosphonates: implications for osteoporosis management. Mayo Clin Proc 2009;84:632-7; quiz 8.

39 Salari P, Abdollahi M. Long term bisphosphonate use in osteoporotic patients; a step forward, two steps back. J Pharm Pharm Sci 2012;15:305-17.

40 Yun H, Curtis JR, Guo L, et al. Patterns and predictors of osteoporosis medication discontinuation and switching among Medicare beneficiaries. BMC Musculoskelet Disord 2014;15:112.

41 Tosteson AN, Do TP, Wade SW, et al. Persistence and switching patterns among women with varied osteoporosis medication histories: 12-month results from POSSIBLE US. Osteoporos Int 2010;21:1769-80.

42 Mechanic D, Tanner J. Vulnerable people, groups, and populations: societal view. Health Aff (Millwood) 2007;26:1220-30.

43 Adler RA. Osteoporosis in men: a review. Bone Res 2014;2:14001.

44 Modi A, Sajjan S, Gandhi S. Challenges in implementing and maintaining osteoporosis therapy. Int J Womens Health 2014;6:759-69.

45 Willson T, Nelson SD, Newbold J, et al. The clinical epidemiology of male osteoporosis: a review of the recent literature. Clin Epidemiol 2015;7:65-76.

46 Guillemin F, Carruthers E, Li LC. Determinants of MSK health and disability-social determinants of inequities in MSK health. Best Pract Res Clin Rheumatol 2014;28:411-33. 